\title{
The development of a safety training strategy for first and second-year engineering design courses
}

An important aspect of design education in engineering schools is the creation of a physical representation, be that proof of concept of full prototype, to 'close the loop' on the design process. To allow for this hands-on design experience, our fist and second-year design workshops are equipped with various hand and power tools, including drill press, band saw, belt sander and scroll saw. Some difficulties arise in these courses from the general lack of experience many students have in using even basic hand tools. From both an educational and a liability point of view, safety training has become a necessity.

Maintaining student safety while in the lab is a mandatory part of students' design course work. Students must understand the importance of a safe work environment and are individually responsible for following safe practices when using power tools. Historically, each student received a didactic 30-minute overview of the workshop with specific instruction on how to properly and safely use each piece of machinery. Despite the fact that there have been few injuries in the workshops, it became clear through observing students, that a new approach for safety training was needed. However, there was no established program suited for undergraduate students that could be implemented. Therefore, a custom solution was created to address our specific needs. We have developed a comprehensive safety-training program for first- and second-year general engineering students. The focus of this paper is to describe the development, implementation and outcomes of our safety program.

To facilitate the current learning styles of technologically savvy students, safety videos (rather than manuals or instructional lists) were created that could be easily accessed online and viewed by students at a time and place convenient to them. Small hand tools were grouped into a single video module while larger power machines received their own individual video due to the considerable safety focus they require. A standard format and script was written and used throughout to convey the equipment's safety overview, component detail, and proper equipment use. Seven short 4-7 minute detailed informative videos were produced. High quality videography was used for professional results to both maximize the resolution of the close-up detail shots for clarity of the message and to help ensure the students view the modules seriously. Accompanying documentation that mirrors the information in the videos are available on-line and in the workshop. A stand-alone introductory design project was also developed where students are asked to demonstrate safe use of the lab tools to complete a simple construction project. Students will also be expected to pass (with $80 \%$ ) a safety quiz based on the videos and documentation. Lectures focusing on design for safety and safety culture have been created and will be given as part of the courses, providing context to the safety videos. This program's effectiveness will be measured quantitatively and qualitatively. The ultimate goal of this program is to educate students so they carry with them a broader understanding of safety into their remaining years of engineering and into industry. 